\title{
Diabeteskomplikationen
}

\section{Ketoazidosen treffen die Patienten oft „aus heiterem Himmel“}

\author{
Ketoazidosen sind selten und gefürchtet. Unter Dia- \\ betikern liegt die jährliche Inzidenz bei ca. 5-8 Fällen \\ pro 1.000 im Vergleich zu 6-14 pro 100.000 in der \\ Allgemeinbevölkerung. Häufig haben die Patienten \\ das richtige Vorgehen bei einer Ketoazidose noch gar \\ nicht gelernt oder wieder vergessen.
}

Viele Patienten trifft es „aus heiterem Himmel“, so die Erfahrung von Dr. Babette Lorra, niedergelassen in einer diabetologischen Schwerpunktpraxis in Bochum. Rund $20 \%-30 \%$ aller diabetischen Ketoazidosen (DKA) treten vor Erstmanifestation des Diabetes auf und gehen häufig mit einem sogenannten Manifestationskoma einher. Aber auch im Verlauf der Erkrankung können bewusste oder unbewusste Unterbrechung der Insulintherapie, z.B. bei einem Pumpendefekt, Unterdosierung der Therapie oder Infektionen, kardiovaskuläre Komplikationen, Stress, Traumata und Operationen eine Ketoazidose begünstigen, berichtete Lorra.

Zu beachten sich auch Warnungen vor einem erhöhten DKARisiko unter SGLT2-Inhibitoren, die ähnliche Stoffwechelwirkungen wie beim Hungern auslösen können. Auch bei sonst Gesunden kann ein übermäßiger Alkoholabusus, durch den die Glukoneogenese gehemmt wird, oder längeres Fasten zu einer verstärkten Bildung von Ketonkörpern infolge aktivierter Lipolyse und Symptomen einer Ketoazidose führen. „Natürlich haben wir Ketoazidosen in der Praxis", sagte Lorra. Unter ihren Patienten liege der Anteil bei $1 \%$.Neben der Hyperglykämie (Blutzucker $>14$ $\mathrm{mmol} / \mathrm{l})$ sind Übelkeit, Erbrechen, Bauchschmerzen, Dehydrierung, tiefe forcierte Atmung und - je nach Schweregrad - Verwirrung, Bewußtseinstrübung bis hin zum Koma klinische Zeichen einer DKA. Bis zu einer mittelschweren DKA können sich gut geschulte Diabetiker selbst aus der gefährlichen Stoffwechselsituation befreien, berichtete Lorra. Werden aber Gegenmaßnahmen nicht rechtzeitig getroffen, vor allem die rasche Gabe von Insulin und viel Flüssigkeit, können die Patienten auch schnell in einem lebensbedrohlichen Zustand auf der Intensivstation landen.

Die Mortalität der DKA liegt in der Regel vermutlich $<5 \%$, sagte die Diabetologin. Werde rechtzeitig und adäquat behandelt, sinke die Sterblichkeit $<1 \%$. Am wichtigsten ist die frühzeitige Messung von Blutketonen wie BetaHydroxybutyrat.

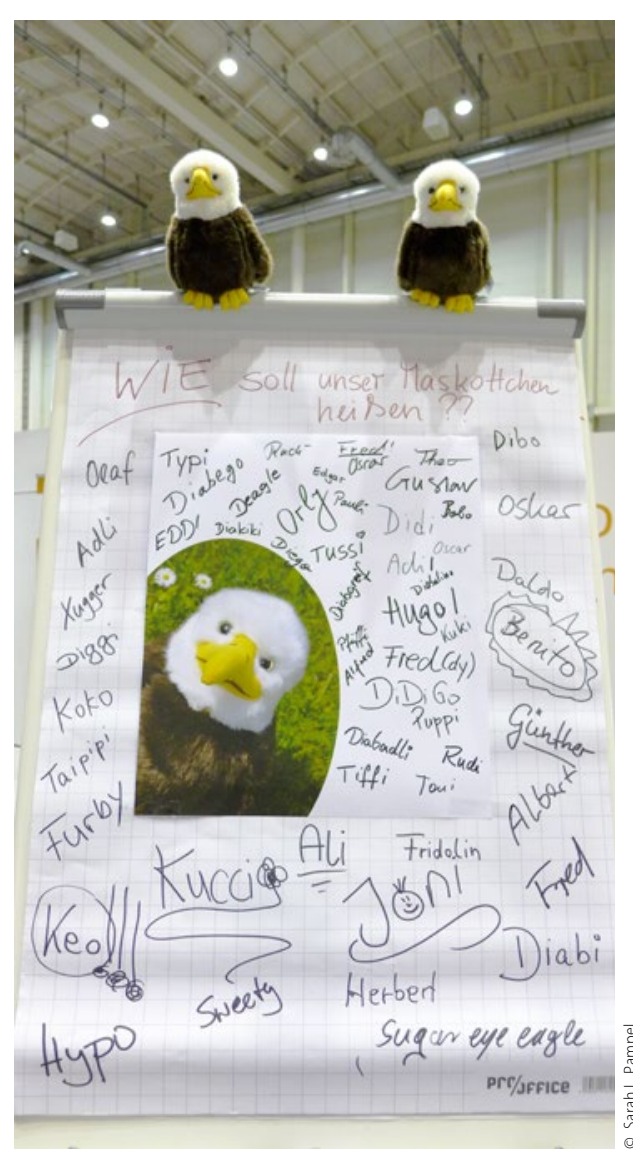

Ebenfalls aus heiterem Himmel: Am DDG-Stand erhielt der Adler letzlich den Namen Albrecht.
Normal sind Werte $<0,6 \mathrm{mmol} / \mathrm{l}$, eine milde Ketaazidose liegt bei Werten von $0,6-1,0 \mathrm{mmol} / \mathrm{l}$, eine mittelschwere bei Werten von $1,0-1,5 \mathrm{mmol} / \mathrm{l}$ vor, bei der der ein geschulter Patient noch selbst Gegenmaßnahmen treffen kann.

Werte $>1,5 \mathrm{mmol} / \mathrm{l}$ erfordern medizinische Hilfe, Werte $>3,0$ $\mathrm{mmol} / \mathrm{l}$ zeigen einen medizinischen Notfall an, sagte Lorra. Auch anhand erhöhter Ketonspiegel im Urin, vor allem Acetoacetat, kann eine DKA nachgewiesen werden. Jedoch sei Urin nicht immer verfügbar und es müsse eine zeitliche Latenz von rund einer Stunde im Vergleich zu den Blutwerten berücksichtigt werden.

Auch in der niedergelassenen Praxis können Patienten mit symptomatischer DKA behandelt werden, wenn sie wach und orientiert sind, so die Einschätzung von Lorra. Allerdings bedeutet dies hohen zeitlichen Aufwand. Studien haben belegt, dass mit einer s.c.-Insulintherapie Stoffwechselparameter wie Glukose-, Beta-Hydroxybutyrat- und Bikarbonatspiegel sowie venöser $\mathrm{pH}$ ähnlich schnell normalisiert werden können wie bei einer i.v.-Therapie.

Lorra schildert beispielhaft den Fall einer 20-jährigen Frau, die sich mit Symptomen wie Durst, Schwindel, Schlappheit, Gewichtsverlust, Amenorrhoe und Pollakisurie zunächst beim Hausarzt vorgestellt hat. Die Laboruntersuchung ergab einen $\mathrm{HbA}_{1 \mathrm{c}}$ von $15 \%$, einen Blutzuckerwert von $256 \mathrm{mg} \%$ und eine deutliche Ketonurie beim Urinstix-Test. Die Patientin wurde in die Schwerpunktpraxis überwiesen, wo sie mit kurz wirksamem Analoginsulin s.c. und ausreichend Flüssigkeit behandelt wurde. Nach 1,5 Stunden hatten sich die Blutglukosewerte und die Ketonurie deutlich verringert. Bei der Patientin wurde ein Typ-1-Diabetes und ein Morbus Basedow neu diagnostiziert. Aufgrund einer thyreotoxischen Krise war sie instabil geworden.

In einer Studie bei 50.000 pädiatrischen Typ-1-Patienten betrug die DKAInzidenz $5 \%$ (Deutschland, Österreich) bis 7 \% (USA). Auch unter einer Insulinpumpentherapie muss mit einer DKA gerechnet werden, berichtete Lorra. Die Inzidenz liegt laut Literaturdaten bei 2 bis 6,6 pro 100 Patientenjahre.

DKA-Patienten mit Bewusstseinstrübungen sowie im Stupor/Koma sollten stationär behandelt werden. Bis zu $10 \%$ der stationären Behandlungen von Diabetikern werden durch DKA verursacht.

Roland Fath

Quelle: AND-Symposium beim Diabeteskongress, 26. Mai 2017 in Hamburg 